\title{
The Concept of "Sociocultural" in the Context of Risks and Threats: The Main Approaches in the Light of the Strategy for Scientific and Technological Development of the Russian Federation*
}

\author{
Nadezhda Bagdasaryan \\ Bauman Moscow State Technical University \\ Lomonosov Moscow State University \\ Moscow, Russia \\ E-mail:ngbagda@mail.ru
}

\author{
Alexandra Yakovleva \\ Lomonosov Moscow State University \\ Moscow, Russia \\ E-mail: afyakovleva@gmail.com
}

\begin{abstract}
The content of the concept "sociocultural" and the concepts of "threat" and "risk" is clarified. It is noted that sociocultural and ideological threats are often subjective, not objective. The classification of social and cultural threats, stipulates that the potential threat of equivalent risk, the real threat to their threats, and updated threat - danger. Presents a picture of research in the field of social and cultural threats and risks, the conclusion is made that the greatest attention is paid to issues such as ethnic and cultural diversity, migration, the problem of identity, cognitive, value, and technological factors affecting the state of society in terms globalization, regionalization, trans-nationalization of conflicts, as well as from the point of view of the formation of civil identity and national consolidation. It is noted that the investigation focused on the definition of resource growth points, such as the stability of the resource, the resource of tolerance, harmony resource in the field already recorded threats, and in recent years a positive trend forecasting planned completely new types of threats and risks that may become relevant already tomorrow.
\end{abstract}

Keywords-sociocultural; scientific and technological development; challenge; threat; identity; conflict

\section{INTRODUCTION}

The strategy of scientific and technological development of the Russian Federation, approved in 2016 by the Presidential Decree and defining the main principles and directions of state policy in the field of science and technology, focuses on freedom of creativity, systemic support, concentration of resources, regional balance, openness, targeting support and fair competition. There is no doubt that it is these key components that can give impetus to the development of the country. Meanwhile, experience shows that the practical implementation of these principles in specific activities faces great difficulties, due to the inadequacy of management mechanisms and the weakness of the methodology for forecasting the development of science

* The article was prepared with the support of the Ministry of Education and Science RF within the framework of the project RFMEFI60117X0018. and technology. Such forecasting will not be effective without identifying possible sociocultural threats and risks. The threats that arise in the socio-cultural sphere, coupled with the solution of a number of very contradictory and extremely complex problems and phenomena in society and culture, can significantly hinder the positive dynamics of the country's development. Identifying and classifying sociocultural threats existing in Russian society requires an appropriate methodological complex that will reveal not only the variety of risks potentially containing threats, but also to make their instrumental assessment. Therefore, it is necessary to clearly understand the parameters of the space that needs to be analyzed in order to identify and classify such threats. In the socio-cultural space, as a complex and open system of human activity, there are many contradictions that have a dual character: the development of these contradictions can become a source of threat, but also serve as a point of growth and development of society. The choice and definition of a methodological approach need to form a conceptual framework for the concepts and categories used in the project. Since the key concept is the concept of "sociocultural", it requires, first of all, a methodological explanation and a sweep. Consider the process of the concept formation through the prism of the problematic of threat assessment and their classification.

\section{CONCEPT "SOCIOCULTURAL"}

We believe it is necessary to clarify the following categories in the context of the tasks being solved in the project: "sociocultural space", "sociocultural sphere", "sociocultural method" (or approach), especially since there are no clear and unambiguous definitions of these categories. The notion of "sociocultural" was formed gradually, beginning with the turn of the nineteenth and twentieth century. The concept of social space has received initial understanding. The fundamental foundation here is the work of Georg Simmel. His reflections on "the qualities of space that allow us to tie ourselves firmly to one or another of its" parts, "but at the same time they must have boundaries" for 
the methodology of our project are important [1].The introduction of culture into space radically changes its characteristics in comparison with the physical and geometric space and gives additional characteristics to the social space. "Social" and "cultural" are considered in indissoluble connection in the framework of the schools P. Sorokin, Yu.M. Lotman, and other researchers which analyzed culture and society in systemic unity. That is, the sociocultural space is not a social + cultural, but a single construct. Here is how, for example, Yu. M. Lotman believes: "the uniqueness of man as a cultural being requires the opposition of his nature to the world, understood as an extracultural space" [2].

Sociocultural space is a multidimensional and multilayered space that semantically constructs its two components: society and culture. The concept of "sociocultural space" has become firmly embedded in the social and humanitarian discourse. However, researchers basically operate on it as intuitively understood for the analysis of various problems by a context beyond which its boundaries are not always discernible. And in fact, the word "space" in itself impresses with limitlessness, although we know how the ideas about it were transformed in the nonclassical, and, moreover, in the post-non-classical science.

Sociocultural space is characterized as qualitative, heterogeneous and non-isotropic, and can be represented in such plans as: values, values and norms; conductors (elements of interaction between individuals, especially in cases where people do not touch each other directly) and human agents. How is it possible to measure this "special set"? Such a measurement is given by "cultural systems and basic singly connected and multiply connected groups along with their subsystems and subgroups", and the number of measurements depends on the accuracy of the social and cultural situation of this socio-cultural phenomenon [3].

Sociocultural sphere is the human dimension of all processes taking place in the state and is the most complex field of human activity. It covers three most important objects: the state, society, personality. In the unity of this integrated system is shown complexity of identifying the risks and threats facing the society and all its constituent segments, continuously changing in the modern world transitive inside (it does not matter, these changes affect the objective or subjective factors). Either directly, or indirectly, they change the configuration and content of each other. Such a dynamic and not always predictable changes upset the balance of the structural order of sociocultural sphere, coherence and complementary in the functioning of its subsystems. That is why the study of the sociocultural sphere requires special method and methodological procedures that are formed within the framework of the sociocultural approach.

Sociocultural approach is primarily systemic methodology, which considers society in the unity of culture and sociality, formed and transformed by human activities [4]. Sociocultural approach - node, the central consideration in vulnerability and threat - he seemed to pull together all the components and line threats - at the state level, at the community level and at the level of the individual - in a single unit. His center is a man who in this case appears as an individual, as a socio-cultural unit of humanity.

Within the framework of the sociocultural approach, a theoretical and methodological matrix can be used, which is formed on a poly-disciplinary basis, including both the traditional methods of comparative social studies and current ones. In particular, the methods of cognitive sociology, sociolinguistics, symbol sociology, content analysis of discursive practices (diagnosis of the state of cognitive (mental) consensus, or the degree of consistency of beliefs about norms and social fact), etc. Sociocultural approach helps identify the main spheres in which society is associated and culture, ensuring the integrity of society. Thus, the sociocultural dimension is possible as an interdisciplinary one, whose task is to develop criteria and technologies for a multifactor analysis of the consequences of various processes and managerial decisions in conditions of variability and maximum uncertainty of tasks.

\section{ClassificAtion OF SOCIO-CUltural Threats}

Setting the task of classifying sociocultural threats and risks, it is important, along with the concept of "sociocultural", to clarify the concept of "threat" and "risk". Researchers note the vagueness of the scope of the very concept of risk, not to mention the absence of generally accepted threat classifications [5]. Nevertheless, there is a significant amount of both Russian and foreign publications on the problems of identifying and classifying threats. All these publications unite the absence of an exhaustive name and comprehensive classification of these threats, as well as their predominantly descriptive character.

For the purposes of our study, the following classifications are significant:

- Belonging to the source of danger, which forms a threat: external and internal;

- At the level of the existence of threats: at the level of the individual (feelings of alienation, apathy, ...), at the level of society (anomie, growing inequality, sense of social injustice ...), at the level of national culture (destruction of the common value system, negative identity at the basis of national selfdetermination).

Russian sociologists propose to distinguish five main classes of threats associated with: a "hostile environment" (foreign policy threats); with "the crisis of culture and values, the degeneration of the nation"; with "degradation of the economy"; with "anti-liberal internal politics"; with "spontaneous cataclysms and force majeure" [6]. In the context of sociocultural problems, external threats are largely determined by social and cultural trends emerging at the global level. High research potential in this context has the use of a fan matrix for constructing threat classification [7]. If on the horizontal axis to arrange the spheres of society's life with breakdown into their constituent elements, and along the vertical axis - the corresponding phenomena / practices of social and political life, taking as a basis the 
PESTLE risk classification model [8] (an acronym from Political - Economic - Social - Technological - Legislative Ethical / Ecological), we can divide the specified areas into the following groups:

- P) political - foreign policy, internal political and regulatory;

- E) economic - economic and financial;

- S) social - demographic, medicine and health, societal, education and science, psychological;

- T) technological - technological;

- L) ethical - spiritual and moral sphere;

- E) ecological - ecological.

Accordingly, at the intersection of each of the axes, a specific threat area is formed. This approach to classification allows us to give our working definition of a threat as a result of the negative impact of specific factors on various spheres of society's life. Upon completion of an exhaustive list of threats, another way of classifying may be to determine whether the threat is on the political agenda (defined by law, present in the official political discourse) and whether it is understood by society (recorded in public opinion polls) by assigning binary labels (yes / no), which will reveal the "invisible" level of threats. In accordance with the elaborated scale by ranking the intensity of attention of society and authorities, each of the above threats determines the qualitative component of the assigned values. In addition, it is possible to classify sociocultural threats according to the degree of their current actualization and the expected dynamics: potential threats; real threats; actual threats. The current actualization of threats, including early detection and forecasting in one form or another, should become one of the tasks of the scientific community within the framework of the Strategy. This task should contribute to the development of systemic solutions to support those research priorities in their interrelation with the challenges and threats on which a request is formed in society and the state and which can represent potential threat points or determine the need for new scientific knowledge to ensure their competitiveness in medium and long term, to form growth points in these spheres.

\section{PROBLEMS OF SOCIO-CUltural ThreAtS IN THE ECOSYSTEM OF RUSSIAN SCIENCE}

Each new stage of technology development generates new types of risks and dangers, which, as a rule, are not visible at the stage of their creation. Engineering and technical thought focuses on building up, but not on the calculation of possible risks. We note this as a special problem requiring intervention in the system of training of engineering personnel [9]. Here, focusing on the duality of the trajectories of technological development, we will set as a promising (and so far, hard-to-implement) task of preventing and reformatting scientific and technological development from "threat points" to "growth points". Calculation and elimination of risks on the basis of long-term forecasting of technological development require its inclusion in the socio-cultural context: the knowledge and technologies obtained should be useful to society, and authorities, and science itself. It should be noted that against the backdrop of rapid development of science and technology, issues related to the role of the scientific community and science in society, with the social role of science and its own state, are becoming more relevant. Due to the development of its social function, science is increasingly adapting to the needs and requirements of the society, which has its positive and negative consequences associated with the development of priorities, funding, and a comprehensive evaluation of scientific activity. To characterize the current state of the Russian science sector in the field of socio-cultural threats, it is necessary to identify the main problem clusters within which the most active research is conducted, and to determine their significance and place in the ecosystem of Russian science as a whole. We believe that it is specifically problem-oriented research that is today a field within the sphere of production of scientific and technical knowledge, which corresponds to the social demand as much as possible.

A complex of sociocultural challenges and threats can be considered in several spheres: socio-psychological, political, ideological and cultural. It should be noted that mainly modern research formulates the research problem through the strategy of structural and functional analysis of the processes of transformation of the state of society and the identification of risk factors in a particular research field. Also, in addition to identifying the factors that ensure the preservation of stability and increase the degree of consolidation of society, the work is aimed at determining the resources of growth points: researchers assess the resource of stability, the resource of tolerance, the resource of consent, etc. It is important to note that it is the transformation of the socio-cultural sphere and the reaction of the scientific community to public inquiries that largely shapes the research space in this field, which distinguishes socio-humanitarian knowledge from other scientific branches. For example, scientists note that the "ethnic revival", the strengthening of nationalist movements, the periodic aggravation of interethnic contradictions, the negative consequences of poorly controlled migration - required the scientific community to respond to public inquiries" [10]. Such a scientific strategy demonstrates one important fact: researchers mostly seek in the field of risk points of growth and stability and overcome potential, but already fixed threats.

Studies of socio-cultural problems that Russian scientists have been carrying out in recent years can be divided into several major problem clusters, which can be conditionally reduced to sociocultural conflicts (especially due to cultural complexity and ethnic diversity), consolidation and adaptation of society. In fact, it is in the sphere of conflicts of different nature, consolidation and adaptation of society that most of the previously identified threats in the classification are fixed. The scientists note the important problem of the lack of a verified methodological and comparative complex for the analysis, assessment and forecasting of risks that can produce interethnic conflicts and impede consolidation and adaptation and consider it necessary to create an interdisciplinary tool for measuring ethnic conflict, making it the object of systematic scientific analysis [11]. 
Researchers introduce indices of social well-being, which allow to predict the degree of conflict. Regulation of the labor market, modernization of the region-center relations, development of the tools of the territories and local communities are highlighted as the main factors that allow reducing the threat of conflict. These conclusions should seriously transform the agenda of public policy in the field of interethnic relations, which can ensure the interaction of cultures and the dialogue of worldviews, the maintenance of a common sociocultural space in a Russian multinational, multicultural, poly-confessional society, combine political practices and scientific results in the field of social security, civil self-consciousness and civil identity, consolidation of the Russian civil nation. One of the key factors distinguishes the factor of state-civic identity as the most important indicator of the unification of society, its integration, which, taking into account regional, cultural, political, historical and other peculiarities, allows determining the resource of interethnic harmony in the consolidation of society. Positive and negative attitudes, the potential for consolidation of various ethnic groups of citizens, as well as the problem of the adaptation of migrants integrated into these societies, are studied in terms of the potential of interethnic harmony. Interethnic harmony is interpreted as a "multi-component" social disposition, which includes various components, among which the usual orientations of Russians play an important role [12] [13].

It is revealed that along with the obvious causes and factors of the formation of such risk zones, there are a lot of latent, hidden reasons why a set of factors provoking a critical situation can form in one or another sphere. This is primarily cognitive factors, linguistic and cognitive models, which include discourse practices, socio-cultural concepts, myths, forms and methods of speech and network communication, as well as various social technologies, that is, everything that constructs a sociocultural field. Both the experts, the expert community and ordinary citizens involved in intensive information exchange need navigation technologies in the communicative space in order to identify the social dangers and risks manifested in various discourses, as well as algorithms for critical analysis of texts, estimates of cognitive reactions used in them and discursive strategies. The identification of the mechanisms for the formation of certain myths and stable images, generated as a result of information exchange and various forms of discursive manifestation, forming, for example, the policy of memory, also makes it possible to identify both real and imaginary threats in the socio-cultural and ideological spheres [14] [15] [16].

\section{CONCLUSION}

So, the content of the concept "sociocultural" and the concepts of "threat" and "risk" is clarified. The characteristics of the concepts "sociocultural space", "sociocultural sphere", "sociocultural approach" are defined. It is noted that sociocultural and ideological threats are often subjective, not objective. A version of the classification of sociocultural threats is presented, it is fixed that potential threats are equivalent to risks, real threats to threats themselves, and actual threats are dangers. A picture of research in the field of social and cultural threats and risks was presented, the conclusion is made that the greatest attention is paid to issues such as ethnic and cultural diversity, migration, the problem of identity, cognitive, value, and technological factors affecting the state of society in terms globalization, regionalization, trans-nationalization of conflicts, as well as from the point of view of the formation of civil identity and national consolidation. It is noted that the investigation focused on the definition of resource growth points, such as the stability of the resource, the resource of tolerance, harmony resource in the field already recorded threats, and in recent years a positive trend forecasting planned completely new types of threats and risks that may become relevant already tomorrow.

\section{REFERENCES}

[1] G. Simmel, Selected. Volume 1\&2. M.: Yurist, 1996. V. 2. Contemplate of life. 607 p., p. 15

[2] Yu. M. Lotman, Semiosphere: Culture and Explosion. Inside minded worlds. Articles. Research. Notes. SPb., 2000. 704 p. P. 21.

[3] P. A. Sorokin, A System of General Sociology. Moscow: Astrel, 2008

[4] N.I. Lapin, Socio-cultural Approach and Societal Functional Structures // Sociological Studies. no. 7. 2000, p. 7.

[5] Analytical report of the Institute of Sociology RAS "National Security of Russia in expert assessments". M., 2012.

[6] Ibid.

[7] S.G. Kordonsky, Classification and ranking of threats // Domestic notes. 2013. 2 (53)

[8] Paul Hopkins, Fundamentals of Risk Management, Understanding, evaluating and implementing effective risk management. Kogan Page, 2017.

[9] N.G. Bagdasaryan, V.S. Koshik, NBIK-technologies as a challenge to education / / Humanitarian Bulletin Bauman MSTU no. 1 (63). 2018. http://hmbul.ru/issues/63.html

[10] Ethnological monitoring: The development of fundamental approaches to ethno-political monitoring for the optimization of interethnic relations and the prevention of conflicts in Russia "// Ed. M.Yu. Martynova, V.V. Stepanova. Moscow: IEA RAS, 2017.

[11] Ethno-political situation in Russia and neighboring countries in 2014. Annual Report of the Network for Ethnological Monitoring and Early Warning of Conflict / Ed. V.A. Tishkov and V.V. Stepanov. Annual Report of the Network for Ethnological Monitoring and Early Warning of Conflict / Ed. V.A. Tishkov and V.V. Stepanov. Moscow: IEA RAS, 2015, pp.195-199.

[12] L.M. Drobizheva, Yu.V. Harutyunyan, The ways passed and some problems of modern Russian ethno-sociology // Sociological Studies. 2017. no. 7 (363), pp. 102-112.

[13] I.S. Semenenko, Identity politics and identity in politics: ethnonational perspectives, European context // Polis. Political Studies, 2016. no. 4, pp. 8-28.

[14] A.I. Miller, The politics of memory in post-communist Europe and its impact on the European memory culture // Politiya: Analysis. The Chronicle. Forecast (Journal of Political Philosophy and Sociology of Politics). 2016. no. 1 (80), pp. 111-121.

[15] Methods of cognitive analysis of word semantics: computer-hull approach / under general. Ed. V. I. Zabotkina. M .: Languages of Slavic Culture, 2015. 344 p.

[16] N.G. Bagdasaryan, Memory Policy: the Past as a Product of Interpretation / Philosophy of Revolutions and Global Trends of Modernity / Proceedings of the International Scientific Conference (Dubna, April 14, 2017) / Ed. N.G. Bagdasaryan, V.G. Fedotova. Dubna, 2017, pp. 5 - 13. 